\title{
Patterning Poly(3-Hexylthiophene) in the Sub-50-nm Region by Nanoimprint Lithography
}

\author{
Giuseppe Scarpa, Alaa Abdellah, Armin Exner, Stefan Harrer, Guillermo Penso Blanco, Wolfgang Wiedemann, \\ Lukas Schmidt-Mende, and Paolo Lugli, Senior Member, IEEE
}

\begin{abstract}
We use thermal and room temperature nanoimprint lithography (NIL) for directly patterning the photoactive polymer poly(3-hexylthiophene-2,5-diyl) (P3HT) in the sub-50-nm region. Different types of molds were used to directly imprint the desired structures into P3HT thin films. Good pattern transfer is achieved independent of the presence of other underlying polymer layers or the type of substrate incorporated. Further, we discuss the future application of this technology to the fabrication of ordered heterojuction organic photovoltaic devices and demonstrate that the NIL process involved does not damage the polymer or alter its chemical or electrical properties.
\end{abstract}

Index Terms-Nanoimprint lithography (NIL), nanostructuring, organic photovoltaic devices (OPVs), organic semiconductors, poly(3-hexylthiophene-2, 5-diyl) (P3HT).

\section{INTRODUCTION}

$\mathbf{E}$ LECTRONIC and optoelectronic devices based on organic semiconductors are increasingly attracting the attention of a steadily growing number of industrial and academic research groups, driven by their potential for low-cost applications with relaxed constraints with respect to their inorganic counterpart [1]-[4]. Of special interest are the devices based on solution processable polymeric materials, and among those, regioregular poly(3-hexylthiophene) (P3HT) is one of the most promising conducting polymers with a relatively high electrical conductivity and nonlinear optical properties [5], [6]. Being able to pattern organic materials in the micrometer range and below is essential: for electronic devices, an improvement of device performances in terms of operations speed can be achieved, whereas for optoelectronic devices, the interaction between matter and light at the nanometer scale can be further exploited [7], [8]. Unfortunately, conventional techniques for achieving micrometeror submicrometer-sized structures are not suitable or present

Manuscript received July 2, 2008; revised January 20, 2010; accepted March 30, 2010. Date of publication April 19, 2010; date of current version May 11, 2011. This work was supported by the cluster of excellence Nanosystems Initiative Munich. The work of S. Harrer was supported by the Presidential Science and Engineering Research Fellowship of the Karl-Max-von-Bauernfeind Verein e.V., Technische Universität München. The review of this paper was arranged by Associate Editor B. Yu.

G. Scarpa, A. Abdellah, A. Exner, S. Harrer, G. Penso Blanco, and P. Lugli are with the Institute for Nanoelectronics, Departement of Electrical Engineering and Information Technology, Technische Universität München, 80333 Munich, Germany (e-mail: scarpa@tum.de).

W. Wiedemann and L. Schmidt-Mende are with the Hybrid Nanostructures Group, Department of Physics and Center for NanoScience, LudwigMaximilians University Munich, 80799 Munich, Germany.

Color versions of one or more of the figures in this paper are available online at http://ieeexplore.ieee.org.

Digital Object Identifier 10.1109/TNANO.2010.2048433 some limitations when applied to organic semiconductors. Different approaches for transferring relief structures into organic materials, for example, are screen printing [9], [10], ink-jet printing [11], [12], soft lithography [13], [14], and nanoimprint lithography (NIL) [15]. Austin and Chou [16] applied nanoimprint techniques for fabricating 70-nm channel P3HT organic thin-film transistors, but rather than directly patterning the semiconductor, they performed the lithographic step on the $\mathrm{SiO}_{2}$ constituting the gate oxide on the $\mathrm{Si}$ substrate. Recently, two direct patterning techniques have been applied to P3HT layers. The first one used micromolding in capillaries with ice molds [17], [18], while the second one performed nanoindentation lithography on a scanning probe microscope (SPM) platform [19]. It is nevertheless difficult to imagine an extension of such special techniques to those practical applications requiring large-area low-cost compatible fabrication technologies. NIL has also been successfully applied by Kim et al. [20] for generating surface-relief gratings on P3HT and fullerene blends, which lead to improved performance of organic solar cells. Since NIL is amenable to low-cost large-area roll-to-roll fabrication, the latter example shows the great potential of this technology for a wide range of important engineering applications.

In this study, we demonstrate thermal (see Section II) and room temperature (see Section III) NIL for directly patterning P3HT films with feature sizes down to $20 \mathrm{~nm}$. For accomplishing this result, molds fabricated by molecular beam epitaxy (MBEmolds) have been used. Finally, in Section IV, we conclude with a discussion and summary of key results and future plans.

\section{Structuring P3HT BY Thermal NIL}

\section{A. Materials and Methods}

We used a commercially available state-of-the-art NIL tool (Obducat 2.5-in equipment, Obducat, Sweden) for structuring the material of interest. The structures imprinted into the polymer were defined using commercially available 2-in-diameter $\mathrm{Ni}$ and $\mathrm{Si} / \mathrm{SiO}_{2}$ molds (NILT Technology, Denmark) comprising approximately 100 -nm-deep line gratings of various lateral feature sizes ranging between $35 \mathrm{~nm}$ and $300 \mathrm{~nm}$. Further, porous alumina templates featuring an average pore width as well as pore-to-pore distance of approximately $60 \mathrm{~nm}$, which were fabricated by a collaboration partner, were used as molds for obtaining 2-D pillar structures. The samples to be imprinted were placed on the bottom plate of a gas-tight heatable as well as coolable chamber of the NIL tool with the mold manually placed upside down onto the imprint polymer layer. The imprint step was performed by pumping compressed air into the upper 
section of the chamber pressing a metal foil (posed on top of the mold/substrate sandwich structure) and the mold itself down against the substrate. Thus, mold features were transferred into the polymer layer. Finally, after cooling of the substrate, the mold was released from the imprint polymer layer. Imprint duration, temperature, and pressure were computer controlled, in situ monitored, and could be preset.

The imprint polymer chosen was a highly regioregular (90\%93\%) P3HT purchased from Rieke Metals, Inc. used without any further purification. The nominal molecular weight (Mw) is specified to be around 50000. Being a comb-like stiff polymer with flexible hexyl side chains, P3HT cannot be strictly considered a glassy polymer, like the ones conventionally used for thermal imprinting. This particular configuration leads to a semicrystalline structure, whose morphology and orientation also depend on the method used for preparing the film, like solvent casting or spin coating. In particular, the orientation in spin-coated films depends on both regioregularity and $\mathrm{Mw}$ [21]. Studies of the thermodynamic phase behavior based on calorimetric measurements revealed a phase transition from a crystalline to a layered liquid crystalline state at $210^{\circ} \mathrm{C}-225^{\circ} \mathrm{C}$ in regioregular $\mathrm{P} 3 \mathrm{HT}$, and a glass transition temperature at $-3{ }^{\circ} \mathrm{C}$ in regiorandom $\mathrm{P} 3 \mathrm{HT}$ [22]. Thus, the phase transition temperature depends on the extent of the regioregularity of the material used and it is not surprising to find in the literature very broad order-disorder phase transitions in a temperature range well below $200^{\circ} \mathrm{C}$, where hysteresis are also present [23], [24].

\section{B. Results and Discussion}

The first step toward achieving proper pattern transfer is finding the optimum imprint parameters for the given polymer. To determine the best imprint temperature, a series of samples were imprinted with different temperatures while fixing pressure and time to 70 bar and $300 \mathrm{~s}$, respectively. The same procedure was then repeated to determine the best imprint pressure; this time, however, samples were imprinted using different pressures while fixing temperature and time to $125^{\circ} \mathrm{C}$ and $300 \mathrm{~s}$, respectively. All samples were prepared by depositing P3HT from a dichlorobenzene-based solution of $2 \mathrm{wt} \%$ concentration. The P3HT layers were deposited onto cleaned Si substrates by spin coating the samples at $1000 \mathrm{r} / \mathrm{min}$ for $90 \mathrm{~s}$, resulting in a layer thickness of approximately $100 \mathrm{~nm}$. No thermal treatment of the samples was performed prior to imprint. The mold used for imprint comprised a line grating with 250 -nm-sized features. This relatively large feature size was chosen during optimization to facilitate the characterization of the samples. The characterization of the imprinted samples was performed using atomic force microscopy (AFM). Imprint depth, measured from a line profile of the AFM scan, was selected to be the main criteria for judging imprint quality. Fig. 1(a) shows a section of an AFM scan of one of the samples $\left(T=125^{\circ} \mathrm{C}, P=70\right.$ bar, $t=$ $300 \mathrm{~s}$ ) and the corresponding line profile indicating the imprint depth mentioned earlier. Further, the graph plotted in Fig. 1(b) presents the imprint depths measured for different samples versus the imprint temperature. The inset shows a second graph, where the imprint depths are plotted versus imprint pressure. In
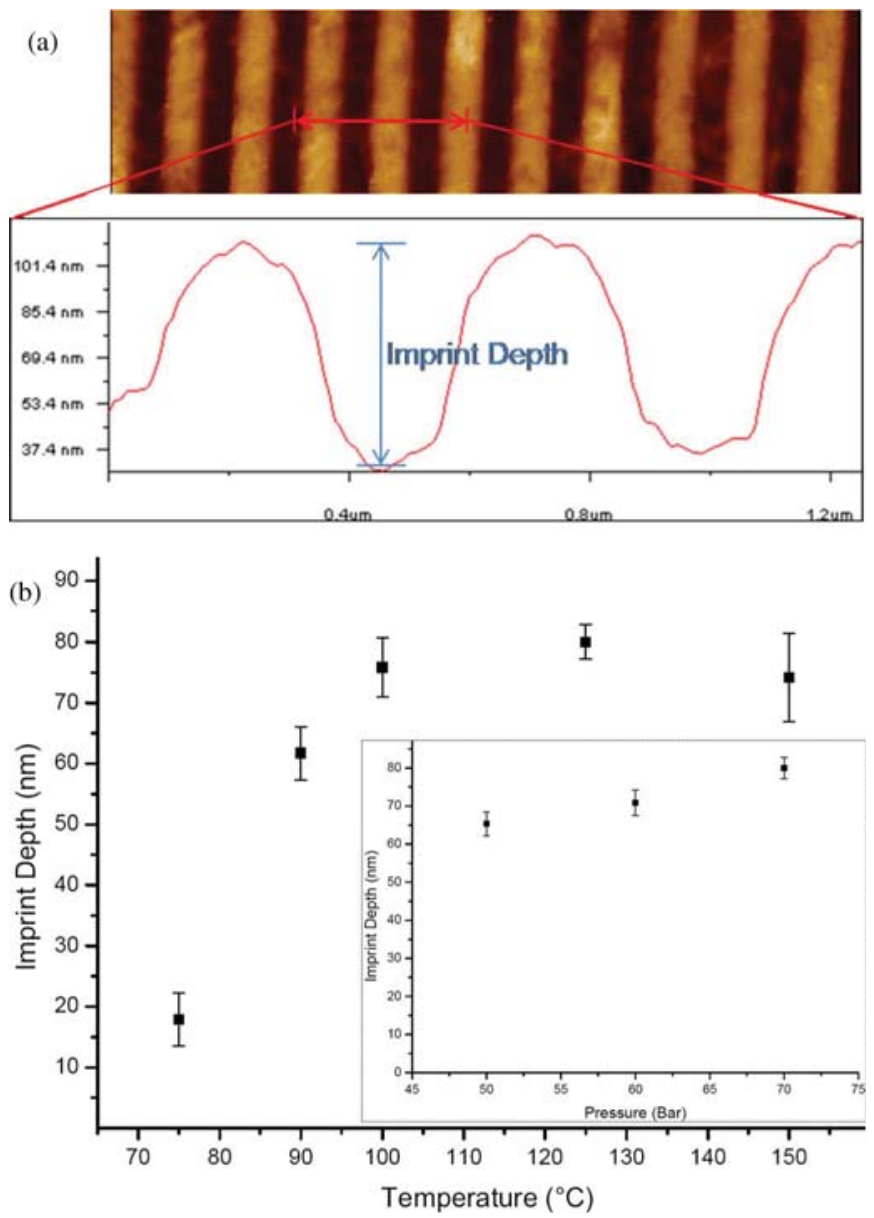

Fig. 1. Section of an AFM scan of a P3HT/Si sample imprinted at $125^{\circ} \mathrm{C}$ and 70 bar for $300 \mathrm{~s}$, and the corresponding line profile indicating the imprint depth used for comparison (top). Graph presenting the imprint depth measured for different samples plotted against imprint temperature as well as against imprint pressure (bottom).

both cases, several measurements were taken at different areas for each sample. The results indicate that the optimum imprint temperature for the purchased material lies between $100^{\circ} \mathrm{C}$ and $125^{\circ} \mathrm{C}$. Although the sample imprinted at $150^{\circ} \mathrm{C}$ showed only a slightly lower average imprint depth, the pattern transferred was of lower quality, which can also be seen in the higher deviation (larger error bar) between the different measurements at that point. This is probably caused by parts of the polymer sticking to the mold while imprinting at more elevated temperatures. On the other hand, the relation between imprint depth and imprint pressure shows a less interesting behavior, which is nearly linear in the examined region. However, it reveals that reasonable pattern transfer can already be achieved at a pressure of 50 bar.

Using the results obtained from the experiments discussed earlier, we attempted to structure P3HT down to the sub-50$\mathrm{nm}$ region. For this purpose, a $\mathrm{Si} / \mathrm{SiO}_{2}$ mold comprising a line grating with 35-nm feature sizes over an area of approximately $1 \mathrm{~cm}^{2}$ was used. The imprint was performed at a temperature of $120^{\circ} \mathrm{C}$ and a pressure of 70 bar for 300 s. Fig. 2 shows an AFM topography image of the imprinted sample scanned over an area of $4 \mu \mathrm{m} \times 4 \mu \mathrm{m}$. The line pattern transferred to the 


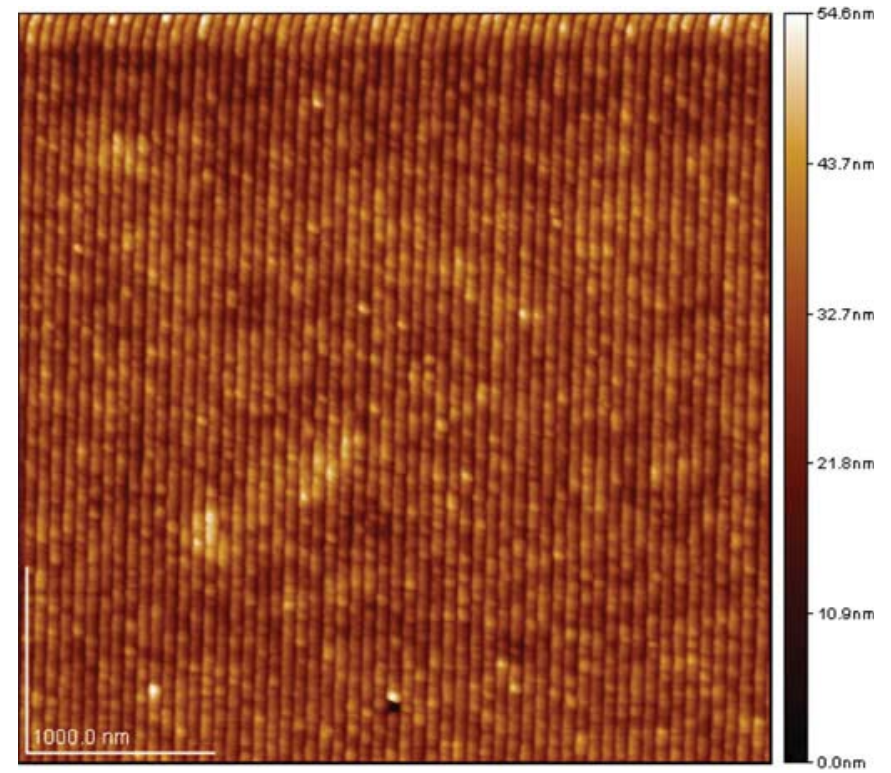

Fig. 2. AFM topography image of an imprinted sample scanned over an area of $4 \mu \mathrm{m} \times 4 \mu \mathrm{m}$. The imprint was performed on a $\mathrm{P} 3 \mathrm{HT} / \mathrm{Si}$ sample at $120^{\circ} \mathrm{C}$ and 70 bar for $300 \mathrm{~s}$ using a $\mathrm{Si} / \mathrm{SiO}_{2}$ mold featuring a $35-\mathrm{nm}$ line grating.

P3HT layer is clearly observed and single lines can be easily distinguished. Note that all AFM images shown here were taken in tapping mode using a standard SPM tip. This is the reason why the lines appear to be touching each other with trenches in between a depth and width smaller than expected. For a more accurate depth measurement of such small structures, an ultrasharp high-aspect-ratio SPM tip is needed. Despite this issue, random scans over the imprinted area proved the imprint process to be suitable for imprinting larger areas of $\mathrm{P} 3 \mathrm{HT}$, which is of significant importance for its future application to active organic devices.

The most promising application of nanostructured P3HT layers is related to organic photovoltaic devices (OPVs) and is going to be discussed in more detail in Section IV. For this application, it is of great interest to increase the surface area of the P3HT layer as much as possible. Hence, 2-D nanostructures would be more favorable for increasing the surface area even further. In Fig. 3 (top), a 3-D AFM image is shown, which demonstrates successful pattern transfer of a 2-D pillar structure imprinted using the porous alumina templates described earlier. As in the earlier case, reliable pattern transfer is achieved over the entire structured area $\left(\sim 1 \mathrm{~cm}^{2}\right)$ with high uniformity as indicated by the $4 \mu \mathrm{m} \times 4 \mu \mathrm{m}$ AFM scan shown. Characterizing a smaller area of $1 \mu \mathrm{m} \times 1 \mu \mathrm{m}$ by AFM, shown in the bottom-left image of Fig. 3, visualizes the individual pillars of around $60 \mathrm{~nm}$ diameter. As for the 35-nm line structure, due to the dimensions of the SPM tip used, the spacing between the pillars appears narrower than expected. This becomes clearer when examining such a structure using an SEM, as shown in the bottom-right image of Fig. 3, where the spacing between pillars appears wider.

It is important to mention that similarly good imprint results were obtained when imprinting P3HT layers deposited onto plain glass, indium tin oxide (ITO)/glass, and

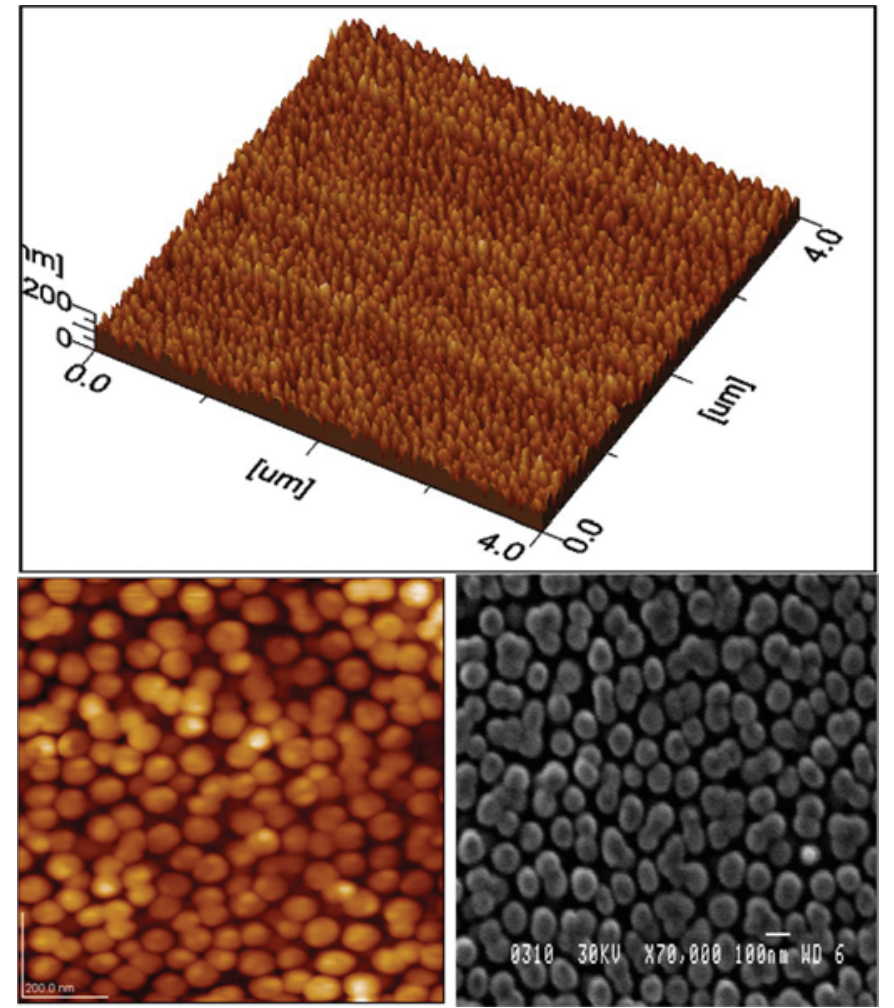

Fig. 3. 3-D AFM image demonstrating successful pattern transfer of a 2-D pillar structure imprinted using the porous alumina templates with $60 \mathrm{~nm}$ average pore width (top). Characterization of a smaller area of approximately $1 \mu \mathrm{m} \times 1 \mu \mathrm{m}$ by AFM (bottom left) and SEM (bottom right).

Poly(3,4-ethylenedioxythiophene) poly(styrenesulfonate) (PEDOT:PSS)/ITO/glass substrates using the different types of molds. Being able to structure P3HT layers independent of the underlying layers and type of substrate is essential for applying this technology to organic electronic and optoelectronic devices.

\section{Structuring P3HT By RoOM TEMPERATURE NIL}

\section{A. Materials and Methods}

We investigated the potential of MBE room temperature NIL (MBE-RTNIL) [25] for patterning P3HT. RTNIL has two core advantages with respect to classical thermal NIL: 1) it can be used to pattern electrically, optically, or magnetically active polymer materials that may change or even lose their distinct functionality upon heating in thermal NIL processes or exposure to radiation in conventional optical, e-beam, or UV-curing lithography and 2) it allows for patterning the same area or closely neighboring areas of an imprint polymer layer through sequential steps and repeated NIL cycles. It was, for example, used in conjugated polymer-based photonics [26], and to fabricate oligomer-based organic distributed feedback lasers [27] as well as near-infrared-imprinted distributed feedback lasers [28]. The imprint polymer layer consisted of P3HT (Rieke Metals, Inc.) that was first dissolved in toluene to a concentration of $2 \%$ by weight. We cleaned a silicon wafer in an oxygen plasma asher for $2 \mathrm{~min}$, spin coated the P3HT/toluene solution onto it 
at $1000 \mathrm{r} / \mathrm{min}$, and baked it at $140^{\circ} \mathrm{C}$ for $15 \mathrm{~min}$, resulting in an approximately 100-nm-thick P3HT layer.

Using a positive multiline MBE-mold (we have elaborately described the mold fabrication process elsewhere [25], [29]) and a custom-built RTNIL tool that controllably presses the MBE-mold into a stationary imprint sample applying imprint pressures up to approximately $700 \mathrm{MPa}$ [30], we carried out the following MBE-RTNIL process: 1) imprint sample and MBEmold were mounted in our custom-built MBE-RTNIL tool; 2) a hardened steel plate with an ultraflat surface was mounted between imprint sample and wafer chuck to support homogenous distribution of the force applied by the wafer chuck counteracting the imprint force during the imprint step; and 3) the mold holder unit was operated in such a way that the cylinder force was partly absorbed in the mold holder unit before transferring to the MBE-mold. This buffer function becomes necessary when using positive MBE-molds, since stand-alone positive mold features are highly sensitive to even slightest variations of the imprint pressure. We then performed separate single-step RTNIL experiments at room temperature under atmospheric conditions, applying an imprint pressure of $\sim 350 \mathrm{MPa}(\sim 3.5 \mathrm{kbar})$ for $10 \mathrm{~s}$.

\section{B. Results and Discussion}

Experimental results are shown in Fig. 4. The SEM images show three imprinted grooves, whereas the 21-nm-wide groove was generated by a $19-\mathrm{nm}$-wide line on the mold, the $39-\mathrm{nm}$ wide groove corresponds to a $36-\mathrm{nm}$-wide line on the mold, and the 60-nm-wide groove results from imprinting a 55-nmwide line on the mold [25]. A 3-nm-thick titanium layer was evaporated onto the sample before SEM imaging to prevent charging effects.

The imprint depths in this case were $\sim 20 \mathrm{~nm}$. Sidewall and surface characteristics of imprinted P3HT patterns need to be further improved in order to establish a RTNIL process that is capable of reproducing high-quality sub-50-nm features. However, we could demonstrate that P3HT can be imprinted in the sub-50-nm region at room temperature by performing the developed MBE-RTNIL scheme. Detailed information on latest development efforts of this technology, including process parameter optimization, patterning efficiency, yield data regarding patterning processes, yield optimization techniques, and main potential applications are presented elsewhere [31].

\section{FUTURE APPLICATION TO OPVS}

The results presented here can be particularly appealing for P3HT-based OPVs. In such devices, an exciton (bound electronhole pair) is created upon absorption of a photon. The energy required for the dissociation of such an exciton is introduced by the difference in ionization potentials and electron affinities at the interface between the donor and acceptor materials forming the active layer of the device. In state-of-the-art OPVs, P3HT and the fullerene derivative [6,6]-phenyl C61 butyric acid methyl ester (PCBM) are used as donor and acceptor materials, respectively. Efficient charge-carrier separation requires large interface areas, which are obtained by the nanoscale phase separation in a blend of the two materials. The interpenetrating

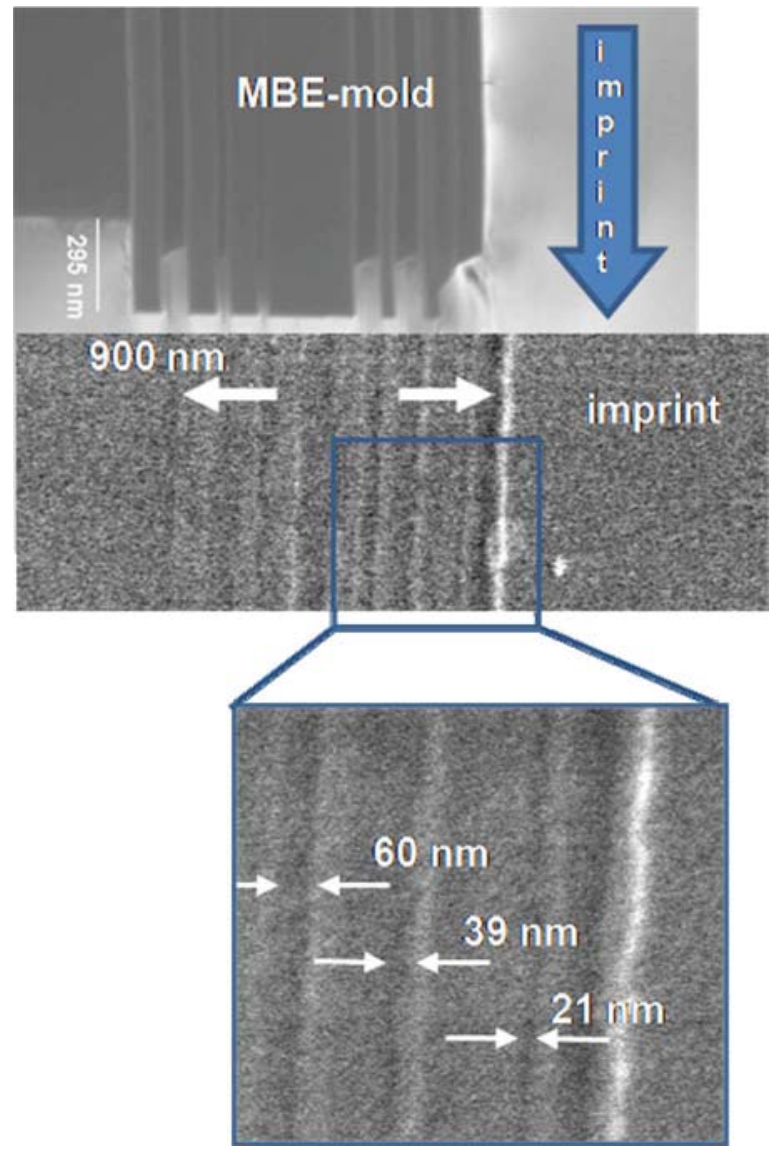

Fig. 4. SEM images of a multiline pattern imprinted onto a P3HT/Si sample at room temperature and an imprint pressure of $\sim 3.5 \mathrm{kbar}$ (bottom) using a positive multiline MBE-mold (top).

network of donor/acceptor interfaces formed during this process has a length scale similar to the exciton diffusion length (in the order of 10 to $20 \mathrm{~nm}$ ), thus allowing dissociation to occur everywhere throughout the bulk before exciton lifetime is reached. The free carriers generated at the interfaces can then be driven to the contacts by the built-in electric field resulting from the different work function electrodes. The improvement in the performance of bulk heterojunction (BHJ) OPVs requires the optimization of all the aforementioned processes, which in turn involves the choice of materials, their combination, the device geometry and structure, and several other parameters. Using nanoimprinting, it should be possible to create controlled acceptor-donor interfaces by patterning P3HT in the sub-50-nm range prior to the deposition of the acceptor material.

However, in order to seriously consider the NIL process described here for the fabrication of ordered heterojunction $(\mathrm{OHJ})$ photovoltaic devices, the compatibility of this process to the imprint polymer (i.e., P3HT) has to be granted. This implies that the process parameters involved in this step of fabrication, such as temperature and pressure applied, do not alter its chemical, optical, and/or electrical properties significantly. Building a complete $\mathrm{OHJ}$ device is, however, rather complicated and still under investigation. The problems that still have to be solved are related to the deposition of the PCBM onto the 


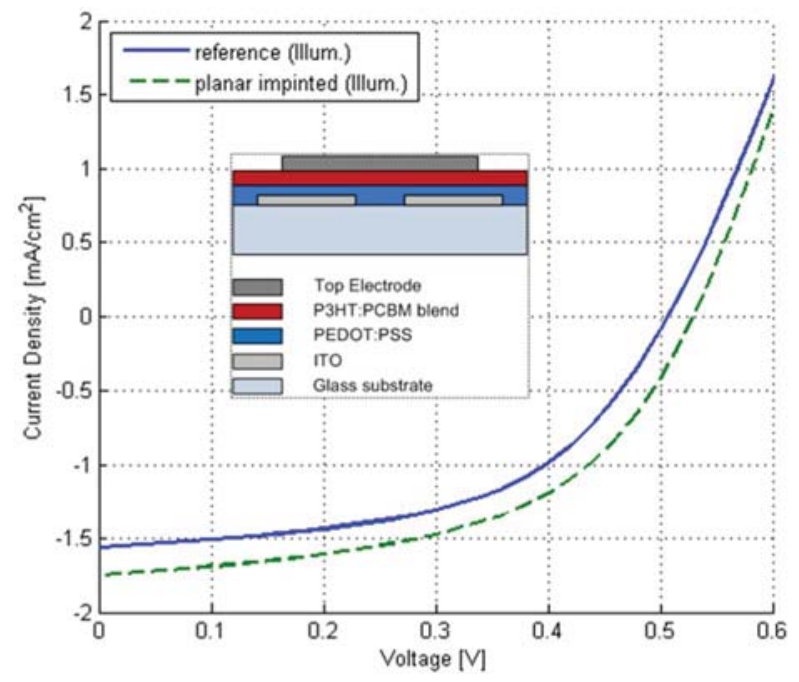

Fig. 5. $I-V$ characteristics comparing a reference device to a planar imprinted device, both measured under illumination with a $100 \mathrm{~mW} / \mathrm{cm}^{2}$ halogen lamp. The inset shows a schematic diagram of the general layout of the $\mathrm{BHJ}$ photovoltaic devices built here.

structured P3HT layer without destroying the pattern while at the same time assuring good filling of the trenches. To overcome this current limitation, we fabricated BHJ devices from a dichlorobenzene-based P3HT:PCBM (1:1) blend solution with 2 wt $\%$ concentration. The active layer was deposited onto a PEDOT:PSS/ITO/glass substrate by spin coating. A reference sample was completed by a thermal annealing process at $80^{\circ} \mathrm{C}$ for $180 \mathrm{~s}$ followed by the evaporation of the metal top electrode. Another sample was imprinted at $80^{\circ} \mathrm{C}$ and 60 bar for $180 \mathrm{~s}$ using a cleaned unstructured $\mathrm{Si}$ wafer. This way, we can assure that both polymers in the blend are exposed to the conditions (i.e., temperature and pressure) of a typical imprint step without being structured, which makes a direct comparison with the reference sample possible. The planar imprinted device was then completed by evaporation of the metal top electrode. A general layout of the BHJ photovoltaic devices prepared here is shown in the inset of Fig. 5.

Fig. 5 shows the $I-V$ characteristics measured for both devices under illumination with a $100 \mathrm{~mW} / \mathrm{cm}^{2}$ halogen lamp. Note that the data plotted is averaged over four diodes for each of the two device types under comparison. This is to ensure that the conclusion taken from the outcome of this experiment represents a general trend. The presented $I-V$ curves clearly demonstrate similar good diode behavior for both devices. Table I compares all key performance parameters extracted from the corresponding $I-V$ curves. Comparable open-circuit voltages and fill factors are calculated for both device types while a $10 \%$ higher short-circuit current is observed for the planar imprinted device, resulting in slightly higher power conversion efficiency. The noticeable enhancement in device performance after imprinting has been observed earlier by Kim et al. for thermally deprotectable polythiophene derivative and was suggested to be due to additional chain-to-chain ordering, resulting from the imprint process [8]. This issue is currently under investigation. From the presented results, it is, however, obvious that
TABLE I

PERFormance PARAMETERS EXTRACTED From the ILluminATED $I-V$ CHARACTERISTICS OF THE FABRICATED DEVICES

\begin{tabular}{l|c|c|c|c|c}
\hline \hline Device & $\begin{array}{c}\mathrm{V}_{\mathrm{oc}} \\
{[\mathrm{V}]}\end{array}$ & $\begin{array}{c}\mathrm{I}_{\mathrm{sc}} \\
{\left[\mathrm{mA} / \mathrm{cm}^{2}\right]}\end{array}$ & $\begin{array}{c}\mathrm{FF} \\
{[\%]}\end{array}$ & $\begin{array}{c}\mathrm{PCE} \\
{[\%]}\end{array}$ & $\begin{array}{c}\mathrm{Rs} \\
{\left[\Omega / \mathrm{cm}^{2}\right]}\end{array}$ \\
\hline Reference & 0.50 & 1.56 & 53.5 & 0.42 & 57.95 \\
\hline $\begin{array}{l}\text { Planar } \\
\text { imprinted }\end{array}$ & 0.52 & 1.75 & 53.1 & 0.48 & 50.79 \\
\hline \hline
\end{tabular}

the device performance is not deteriorated in any way by the imprint step during device fabrication. Hence, we can assume the individual materials, including P3HT, are not damaged by the imprint process.

\section{CONCLUSION}

We demonstrated thermal NIL as well as room temperature NIL using organic semiconducting P3HT films as imprint polymer. Commercially available $\mathrm{Ni}$ and $\mathrm{Si} / \mathrm{SiO}_{2}$ molds as well as custom-built MBE-molds and porous alumina templates enabled imprinting patterns with feature sizes from $100 \mathrm{~nm}$ down to $20 \mathrm{~nm}$. Our experiments revealed good pattern-transfer quality into P3HT layers independent of the presence of other underlying polymer layers or the type of substrate incorporated. Thermal NIL processes were carried out at imprint temperatures around $120^{\circ} \mathrm{C}$, applying a standard imprint pressure of 70 bar for $300 \mathrm{~s}$. RTNIL processes were performed at significantly higher imprint pressures, i.e., approximately $350 \mathrm{MPa}$ (3.5 kbar) applied for $10 \mathrm{~s}$. Samples with multilayer structures (e.g., including PEDOT:PSS) were also produced to demonstrate process compatibility within more complex device architectures in the future. The future application of this technology to the fabrication of OHJ OPVs was discussed in detail. Finally, we proved that the NIL step involved in the fabrication of such kind of devices would not damage the polymer or alter its chemical or electrical properties. Clearly, imprint polymer flow characteristics and the stability of MBE-molds and imprint samples under RTNIL conditions as well as sidewall and surface characteristics of imprinted features have to be further analyzed and optimized. In order to bring the MBE-RTNIL scheme down into the sub-15-nm region, it is necessary to investigate the flow characteristics of P3HT and its capability to fill mold features during the imprint step.

\section{ACKNOWLEDGMENT}

The authors would like to thank S. Strobel and M. Bichler for carrying out and supervising MBE growth process at the WalterSchottky Institute, Technische Universität München. The authors would also like to thank D. Grundler and P. Berberich for granting access to their SEM system.

\section{REFERENCES}

[1] W. Clemens, W. Fix, J. Ficker, A. Knobloch, and A. Ullmann, "From polymer transistors toward printed electronics," J. Mater. Res., vol. 19, pp. 1963-1973, 2004. 
[2] G. Horowitz, "Organic thin film transistors: From theory to real device," J. Mater. Res., vol. 19, pp. 1946-1962, 2004.

[3] C. D. Dimitrakopoulos and D. J. Mascaro, "Organic thin-film transistors: A review of recent advances," IBM J. Res. Dev., vol. 45, pp. 11-27, 2001.

[4] H. Klauk, U. Zschieschang, J. Pflaum, and M. Halik, "Ultralow-power organic complementary circuits," Nature, vol. 445, pp. 745-748, 2007.

[5] H. Sirringhaus, N. Tessler, and R. H. Friend, "Integrated optoelectronic devices based on conjugated polymers," Science, vol. 280, pp. 1741-1744, 1998.

[6] G. Wang, J. Swensen, D. Moses, and A. J. Heeger, "Increased mobility from regioregular poly(3-hexylthiophene) field-effect transistors," $J$. Appl. Phys., vol. 93, no. 10, pp. 6137-6141, 2003.

[7] G. Scarpa, F. Brunetti, S. Harrer, and P. Lugli, "Nanoimprint lithography for optical components," in Proc. 9th IEEE Int. Conf. Transparent Opt. Netw. (ICTON 2007), vol. 2, pp. 194-197.

[8] M. Kim, J. Kim, J. C. Cho, M. Shtein, L. J. Guo, and J. Kim, "Flexible conjugated polymer photovoltaic cells with controlled heterojunctions fabricated using nanoimprint lithography," Appl. Phys. Lett., vol. 90, pp. 123113-1-123113-3, 2007.

[9] H. D. Goldberg, R. B. Brown, D. P. Liu, and M. E. Meyerhoff, "Screen printing: A technology for the batch fabrication of integrated chemicalsensor arrays," Sens. Actuators B, vol. 21, pp. 171-183, 1994.

[10] Z. Bao, Y. Feng, A. Dodabalapur, V. R. Raju, and A. J. Lovinger, "Highperformance plastic transistors fabricated by printing techniques," Chem. Mater. vol. 9, pp. 1299-1301, 1997.

[11] D. Pede, G. Serra, and D. De Rossi, "Microfabrication of conducting polymer devices by ink-jet stereolithography," Mater. Sci. Eng. C, vol. 5, pp. 289-291, 1998 .

[12] T. R. Hebner, C. C. Wu, D. Marcy, M. H. Lu, and J. C. Sturm, "Ink-jet printing of doped polymers for organic light emitting devices," Appl. Phys. Lett., vol. 72, no. 5, pp. 519-521, 1998.

[13] Y. Xia and G. M. Whitesides, "Soft lithography," Аnnu. Rev. Mater. Sci., vol. 28, pp. 153-184, 1998

[14] J. A. Rogers, Z. Bao, A. Makhija, and P. Braun, "Printing process suitable for reel-to-reel production of high-performance organic transistors and circuits," Adv. Mater, vol. 11, no. 9, pp. 741-745, 1999.

[15] S. Y. Chou, "Imprint of sub-25 nm vias and trenches in polymers," Appl. Phys. Lett., vol. 67, pp. 3114-3116, 1995.

[16] M. D. Austin and S. Y. Chou, "Fabrication of $70 \mathrm{~nm}$ channel length polymer organic thin-film transistors using nanoimprint lithography," Appl. Phys. Lett., vol. 81, pp. 4431-4433, 2002.

[17] E. Kim, Y. Xia, and G. M. Whitesides, "Polymer microstructures formed by moulding in capillaries," Nature, vol. 376, pp. 581-584, 1995.

18] X. Yu, R. Xing, S. Luan, Z. Wang, and Y. Han, "Direct micropatterning of polymer materials by ice mold," Appl. Surf. Sci., vol. 252, pp. 8544-8548, 2006.

[19] C. Balocco, A. G. Jones, J. M. Kingsley, J. R. Chan, X. Q. Huang, and A. M. Song, "Scanning probe microscope based nanolithography on conducting polymer films," Jpn. J. Appl. Phys., vol. 45, pp. 2095-2098, 2006.

[20] S. Na, S. Kim, S. Kwon, J. Jo, J. Kim, T. Lee, and D. Kim, "Surface relief gratings on poly (3-hexylthiophene) and fullerene blends for efficient organic solar cells," Appl. Phys. Lett., vol. 91, pp. 173509-1-173509-3, 2007.

[21] H. Sirringhaus, P. J. Brown, R. H. Friend, M. M. Nielsen, K. Bechgaard, B. M. W. Langeveld-Voss, A. J. H. Spiering, R. A. J. Janssen, E. W. Meijer, P. Herwig, and D. M. de Leeuw, "Two-dimensional charge transport in self-organized, high-mobility conjugated polymers," Nature, vol. 401, pp. 685-688, 1999 .

[22] S. Hugger, R. Thomann, T. Heinzel, and T. Thurn-Albrecht, "Semicrystalline morphology in thin films of poly(3-hexylthiophene)," Colloid Polym. Sci., vol. 282, pp. 932-938, 2004.

[23] B. K. Kuila and A. K. Nandi, "Physical, mechanical, and conductivity properties of poly(3-hexylthiophene)-montmorillonite clay nanocomposites produced by the solvent casting method," Macromolecules, vol. 37, pp. 8577-8584, 2004.

[24] M. J. Winokur, D. Spiegel, Y. Kim, S. Hotta, and A. J. Heeger, "Structural and absorption studies of the thermochromic transition in poly(3hexylthiophene),"Synthetic Met., vol. 28, pp. C419-C426, 1989.

[25] S. Harrer, S. Strobel, G. Scarpa, G. Abstreiter, M. Tornow, and P. Lugli, "Room-temperature nanoimprint lithography using molds fabricated by molecular beam epitaxy," IEEE Trans. Nanotechnol., vol. 7, no. 3, pp. 363-370, May 2008.

[26] E. Mele, F. Di Benedetto, L. Persano, R. Cingolani, and D. Pisignano, "Multilevel, room-temperature nanoimprint lithography for conjugated polymer-based photonics," Nano Lett, vol. 5, pp. 1915-1919, 2005.
[27] D. Pisignano, L. Persano, P. Visconti, R. Cingolani, G. Gigli, G. Barbarella, and L. Favaretto, "Oligomer-based organic distributed feedback lasers by room-temperature nanoimprint lithography," Appl. Phys. Lett., vol. 83, pp. $2545-2547,2003$.

[28] P. Del Carro, A. Camposeo, R. Stabile, E. Mele, L. Persano, R. Cingolani, and D. Pisignano, "Near-infrared imprinted distributed feedback lasers," Appl. Phys. Lett., vol. 89, pp. 201105-1-201105-3, 2006.

[29] P. Lugli, S. Harrer, S. Strobel, F. Brunetti, G. Scarpa, M. Tornow, and G. Abstreiter, "Advances in Nanoimprint lithography," in Proc. 7th IEEE Int. Conf. Nanotechnol., 2007, pp. 1179-1184.

[30] S. Harrer, S. Strobel, S. Luber, F. Brunetti, G. Scarpa, M. Tornow, G. Abstreiter, and P. Lugli, International and European Patent Application PCT/EP/2007/004109, 2007.

[31] S. Harrer, S. Strobel, G. Penso Blanco, G. Scarpa, G. Abstreiter, M. Tornow, and P. Lugli, "Technology assessment of a novel highyield lithographic technique for sub-15-nm direct nanotransfer printing of nanogap electrodes," IEEE Trans. Nanotechnol., vol. 8, no. 6, pp. 662670, Nov. 2009

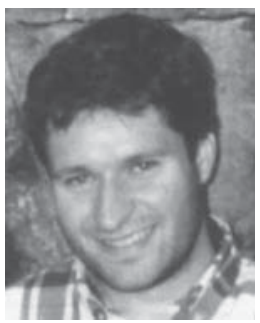

Giuseppe Scarpa received the Laurea degree in electrical engineering from the University of Rome, Tor Vergata, Italy, in 1998, and the Ph.D. degree from Walter Schottky Institute, Technical University of Munich, Munich, Germany, in 2003. His Ph.D. thesis was on design and fabrication of quantum cascade lasers.

$\mathrm{He}$ is currently a Staff Lecturer at the Electrical Engineering Department and a Staff Scientist at the Institute for Nanoelectronics, Technical University of Munich. His research interests include the fabrication of a variety of nanostructures (such organic devices and nanomagnets) and the development of various nanofabrication technologies based on nanoimprint lithography as well as on biosensors and biochips based on organic materials.

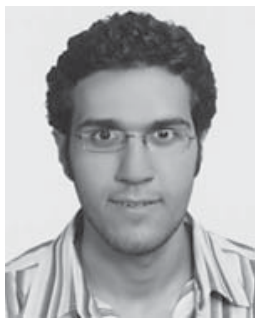

Alaa Abdellah was born in Cairo, Egypt. He received the B.Sc. degree in electronics and communications engineering from the Cairo University, Cairo, in 2005, and the M.Sc. degree in high-frequency engineering and optoelectronics from the Technical University of Munich, Munich, Germany, in 2007, where he is currently working toward the Ph.D. degree at the Institute for Nanoelectronics.

His research interests include fabrication and characterization of organic electronic and optoelectronic on nanoimprint lithography. devices as well as nanofabrication technologies based

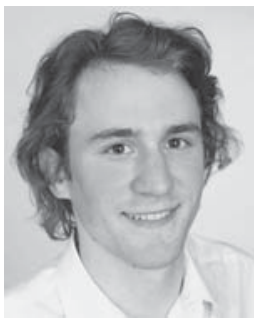

Armin Exner received the Diploma degree in electrical engineering from the Technical University of Munich, Munich, Germany, in 2009, where he is currently working toward the $\mathrm{Ph}$.D. degree at the Institute for Nanoelectronics. His Diploma thesis was on patterning P3HT by nanoimprint lithography.

His research interests include nanostructuring and stamp transfer techniques for optical and chemical detectors. 


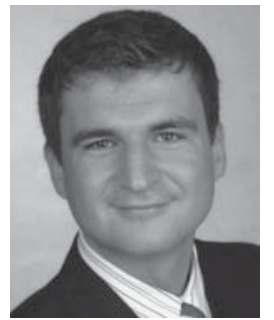

Stefan Harrer received the B.Sc. and Diploma degrees in electrical engineering and computer science from the Technical University of Munich, Munich, Germany, in 2003 and 2004, respectively, where he is currently working toward the Ph.D. degree at the Institute for Nanoelectronics. He also studied Technology Management at the Center for Digital Technology and Management of the Ludwig-Maximilians Universität München, the Technical University of Munich, and the University of California, Berkeley. He received a Masters (Honors) Certificate in Technology Management from the Technical University of Munich and the LudwigMaximilians Universität München, in 2003.

He was a Visiting Graduate Student in the Department of Electrical Engineering and Computer Science, Massachusetts Institute of Technology, Cambridge, during 2005-2006, where he was involved in the research on implementing new nanoimprint lithography techniques. He has authored or coauthored several technical publications, and has two patents pending. His research interests include nanofabrication technologies with special focus on nanoimprint lithography.

Mr. Harrer was the recipient of the IEEE Best Student Paper Award on the 6th IEEE International Conference on Nanotechnology 2006, Cincinnati, OH.

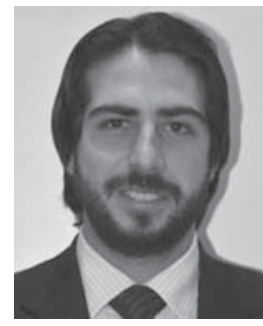

Guillermo Penso Blanco received the B.Sc. degree in telecommunications engineering from the European University of Madrid, Madrid, Spain, in 2008.

$\mathrm{He}$ is currently at the Institute for Nanoelectronics, Technical University of Munich, Munich, Germany, where he was also an Exchange Student from 2006 to 2008 and was involved in the research on sub-15-nm room temperature nanoimprint lithography and nanotransfer printing using MBE-molds. His research interests include nanofabrication techniques with special focus on nanotransfer printing technology.

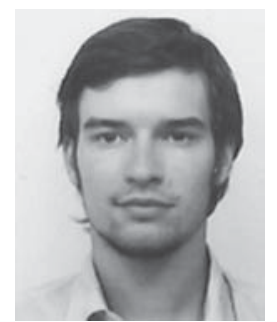

Wolfgang Wiedemann received the Diploma degree in information technology from the Technical University of Munich, Munich, Germany, in 2008. Currently, he is working toward the Ph.D. degree in physics at Ludwig-Maximilians University, Munich, in the group of Prof. Schmidt-Mende.

$\mathrm{He}$ is involved in the research on the influence of the nanomorphology on the device physics in organic solar cells.

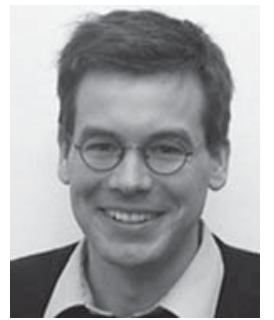

Lukas Schmidt-Mende received the diploma degree in physics from the Ruprecht Karls University of Heidelberg, Heidelberg, Germany, in 1999 and the Ph.D. degree from the University of Cambridge, Department of Physics, Cambridge, U.K., under the supervision of Prof. Sir R. Friend.

In 2003, he joined the group of Prof. M. Grätzel as a Postdoctoral Research Fellow at Ecole Polytechnique Fédérale de Lausanne, Switzerland, where he was involved in the research on solid-state dyesensitized solar cells. In 2005, he rejoined the Department of Materials Science, University of Cambridge, as a Marie Curie Excellence Grant Fellow. He was a University Research Fellow of the Royal Society. In autumn 2007, he joined Ludwig-Maximilians University, Munich, Germany as an Associate Professor in functional nanostructures. His current research interests include the investigation of novel nanostructured materials and hybrid materials for solar cell applications.

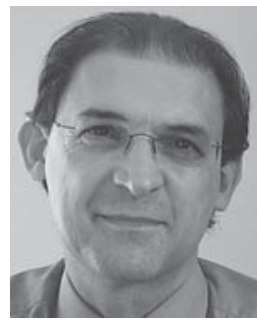

Paolo Lugli (SM'07) received the Laurea degree in physics from the University of Modena, Modena, Italy, in 1979, and the M.Sc. and Ph.D. degrees in electrical engineering from Colorado State University, Fort Collins, in 1982 and 1985, respectively.

In 1985, he joined the Physics Department, University of Modena as a Research Associate. From 1988 to 1993, he was an Associate Professor of solidstate physics at Engineering Faculty, University of Rome Tor Vergata, Rome, Italy, where he became a Full Professor of optoelectronics in 1993. In 2003, he joined the Technical University of Munich, Munich, Germany, where he was appointed Head of the Institute for Nanoelectronics. He has authored more than 250 scientific papers and coauthored the books The Monte Carlo Modeling for Semiconductor Device Simulations (Springer, 1989) and High Speed Optical Communications (Kluwer Academic, 1999). His current research interests include, besides nanoimprint lithography, the modeling, fabrication, and characterization of organic devices for electronics and optoelectronics applications, the design of circuits and architectures for nanostructures and nanodevices, the numerical simulation of microwave semiconductor devices, and the theoretical study of transport processes in nanostructures.

Dr. Lugli was the General Chairman of the IEEE International Conference on Nanotechnology, Munich, in 2004. 